\title{
Interventional Neurorehabilitation for Promoting Functional Recovery Post-Craniotomy: A Proof-of-Concept
}

Anujan Poologaindran ${ }^{1,2}$ MSc, Christos Profyris ${ }^{3,4}$ FC Neurosurg (SA), Isabella M. Young BSc, Nicholas B. Dadario BSc ${ }^{4,5}$, Syed A. Ahsan ${ }^{4}$ BMed, Kassem Chendeb ${ }^{4}$ CS, Robert G. Briggs ${ }^{6}$ MD, Charles Teo ${ }^{4}$ FRACS, Rafael Romero-Garcia ${ }^{1}$ PhD, John Suckling ${ }^{1,2}$ PhD, * Michael E. Sughrue ${ }^{1,4}$ MD

${ }^{1}$ Brain Mapping Unit, Department of Psychiatry, University of Cambridge, Cambridge, UK

${ }^{2}$ The Alan Turing Institute, British Library, London, UK

${ }^{3}$ Netcare Linksfield Hospital, Johannesburg, South Africa

${ }^{4}$ Department of Neurosurgery, Prince of Wales Private Hospital, Sydney, Australia

${ }^{5}$ Rutgers Robert Wood Johnson Medical School, New Brunswick, New Jersey, USA

${ }^{6}$ Department of Neurosurgery, University of Southern California, Los Angeles, California, USA

*Corresponding Author, sughruevs@gmail.com

Corresponding Author: Dr.Michael E. Sughrue

Neurosurgeon, Dept. of Neurosurgery, Prince of Wales Private Hospital, Sydney, Australia

Visiting Researcher, Department of Psychiatry, University of Cambridge, Cambridge, UK sughruevs@gmail.com

P-(02) 83280975 / F-(02) 91696375

Running Title: Interventional Neurorehabilitation

Key Words: connectomics, rehabilitation, functional imaging, transcranial magnetic stimulation, glioma 


\title{
Interventional Neurorehabilitation for Promoting Functional Recovery Post-Craniotomy: A Proof-of-Concept
}

\begin{abstract}
Purpose: The human brain is a highly plastic 'complex' network -it is highly resilient to damage and capable of self-reorganisation after a large perturbation. Clinically, neurological deficits secondary to iatrogenic injury have very few active treatments. New imaging and stimulation technologies, though, offer promising therapeutic avenues to accelerate post-operative recovery trajectories. In this study, we sought to establish the safety profile for 'interventional neurorehabilitation': connectome-based therapeutic brain stimulation to drive cortical reorganisation and promote functional recovery post-craniotomy.
\end{abstract}

Methods: In n=34 glioma patients who experienced post-operative motor or language deficits, we used connectomics to construct single-subject cortical networks. Based on their clinical and connectivity deficit, patients underwent network-specific Transcranial Magnetic Stimulation (TMS) sessions daily over five consecutive days. Patients were then assessed for TMS-related side effects and improvements.

Results: $31 / 34$ (91\%) patients were successfully recruited and enrolled for TMS treatment within two weeks of glioma surgery. No seizures or serious complications occurred during TMS rehabilitation and one-week post-stimulation. Transient headaches were reported in 4/31 patients but improved after a single session. No neurological worsening was observed while a benefit was noted in 28/31 patients post-TMS. We present two clinical vignettes and a video demonstration of interventional neurorehabilitation.

Conclusions: For the first time, we demonstrate the safety profile and ability to recruit, enrol, and complete TMS acutely post-craniotomy in a high seizure risk population. Given the lack of randomisation and controls in this study, prospective randomised sham-controlled stimulation trials are now warranted to establish the efficacy of interventional neurorehabilitation following craniotomy. 


\section{Introduction}

The human brain is a highly plastic 'complex' network [1,2]: it self-organises without a hard blueprint, it adapts to evolving circumstances, and can withstand external insults. Our thoughts and behaviour are directly governed by how our brain networks handle, orchestrate, and execute various internal and external demands [3]. Nevertheless, similar to other naturallyoccurring networks, brain networks can only endure a finite amount of damage before becoming maladaptive and fragmented [4].

The practice of neurosurgery is based on therapeutically altering the brain's global workspace to improve clinical outcomes $[5,6]$. However, since the antiquity of neurosurgery, few strategies have been employed to directly address neurological deficits due to iatrogenic injury. In fact, the usual approach is to send patients to physiotherapy and hope they improve over time in a sufficiently stimulating environment. Moreover, rehabilitation is further complicated when surgical pathology implicates critical areas for motor initiation, alertness, motivation, and consciousness [7]. Furthermore, advanced neurocomputational models suggest the capacity for neuroplasticity greatly varies based on the type of cortical damage which has occurred [8]. Ideally, a fundamental goal of neuro-oncological surgery should be to drive cortical reorganisation and promote functional recovery in the immediate post-operative period. To advance this viewpoint, we coin a new concept called 'interventional neurorehabilitation': connectome-based therapeutic brain stimulation to promote network plasticity and functional recovery. and neurostimulation technologies. Today, state-of-the art connectome methods enable neuroscientists to make highly accurate single-subject predictions on cognition [9-11]. In

28 addition, we are beginning to non-invasively stimulate focally at-depth without perturbing overlaying cortical structures [12]. However, before leveraging the most advanced technologies 
31 sensible. Repetitive transcranial magnetic stimulation (rTMS) is an FDA-approved stimulation 32 therapy routinely performed at hospitals across the world [13]. Given its relative ease and non-

33 invasiveness, the field of TMS has flourished to treat a range of neurological and psychiatric

34 illnesses. In acute and chronic stroke patients, rTMS facilitates cortical reorganization leading to

35 functional preservation or compensation in motor and language abilities [14]. Unfortunately,

36 prognosis is still poor in many these patients, which may be explained by the limited capacity for

37 effective cerebral plasticity following some acute injuries compared to slow growing tumors [8].

38 While meta-analyses highlight the remarkable safety of rTMS in ischemic stroke patients with extremely low-risks for seizures $[15,16]$, there remains limited descriptions on the safety and efficacy of this treatment modality in tumor patients in the acute post-operative period. Given the striking advances in fields outside neuro-oncology, individualised TMS therapy merits investigation to accelerate recovery trajectories post-craniotomy.

In this proof-of-concept study, we sought to establish the safety profile and ability to recruit, enrol, and complete connectome-guided TMS to enhance network plasticity and promote functional recovery following glioma surgery.

\section{Methods}

49 This study was approved by the Human Research Ethics Committee of the South Eastern Sydney Local Health District (SESLHD). Patients provided written informed consent prior to enrolling in our study. All methods were performed in accordance with relevant guidelines and regulations Declaration of Helsinki. The clinical trial was registered at clinicaltrials.gov (NCT03293888).

\section{Patient population}

Patients with supratentorial gliomas who developed a significant post-operative neurological deficit related to motor or language function were invited to take part in an off-label treatment of FDA-approved rTMS. Subjects were only included in this study if TMS was

58 initiated within two weeks post-surgery. Assessments for motor dysfunction were made using the standard Medical Research Council (MRC) 5-point scale ${ }^{[17]}$. To be eligible for rTMS therapy, weakness in an arm or leg needed to be 4-/5 or worse in the hand, proximal arm, foot or proximal 
61 leg at the time of treatment. Language dysfunction was defined using the Aphasia Rapid Test

62 (ART) with a score greater than 3 considered evidence of significant language disturbance ${ }^{[18]}$.

\section{Clinical assessment and Definition of Outcomes}

Neurological assessments were performed immediately prior to treatment with rTMS and one week following the last rTMS session by a blinded team member. Improvement in motor function was defined as grade strength to at least $4+/ 5$ in the affected limb, with either functional hand control or the ability to walk with assistance in the leg. In cases of hemiplegia, improvement in either hand or leg function was considered improvement. Finally, reduction in the patient's pre-treatment ART score by 3 or more points was considered improvement in language.

\section{Connectome-based TMS target selection in neurosurgical patients}

Once recruited, participants underwent a T1-weighted MPRAGE and resting-state fMRI scan. The cortical target was selected based on the patient's primary deficit (i.e. motor or language), our interpretation of any network fragmentation, and our experience with network topology from normative connectomes (i.e. HCP data) ${ }^{[7,19,20]}$.

\section{Imaging Acquisition and Pre-processing Parameters}

The resting-state fMRI was performed on a Phillips 3T Achieva which was acquired as a T2-star EPI sequence, with $3 \times 3 \times 3$-mm voxels, 128 volumes/run, a TE $=27 \mathrm{~ms}$, a TR $=2.8 \mathrm{~s}$, a

82 field of view $=256 \mathrm{~mm}$, a flip angle $=90^{\circ}$ and an 8 minute total run time. Resting-state and diffusion pre-processing was performed using in-house custom machine learning algorithms in Python. Standard image processing steps included skull stripping, motion correction with a 6dimensional rigid body registration, correcting for physiological noise (CompCor), slice time correction, spatial smoothing (6 FWHM Gaussian kernel), high-pass filtering, and co-registration to the patient's structural space ${ }^{1}$. Of critical importance, we do not warp the brain into a standard space like Montreal Neurological Institute (MNI) or Talairach space at any stage of the analyses from patient-specific multi-modal imaging data. 
medRxiv preprint doi: https://doi.org/10.1101/2021.07.27.21260088; this version posted August 4, 2021. The copyright holder for this preprint (which was not certified by peer review) is the author/funder, who has granted medRxiv a license to display the preprint in perpetuity.

All rights reserved. No reuse allowed without permission.

\section{Machine-learning Aided Parcellation for Brain Tumours}

A fundamental challenge for interventional neurorehabilitation post-craniotomy is to

94 apply a parcellation scheme to highly-distorted anatomical brains. The Glasser HCP parcellation

95 scheme is a state-of-the art multi-modal neurobiological division of the cerebral cortex ${ }^{[21]}$.

96 However, it was not designed to be applied to brains with large lesions and oedema. We aimed to

97 directly address this challenge by determining new HCP parcellation locations by using a

98 proprietary machine learning algorithm (Omniscient Technologies) -Figure 1 is the connectome construction pipeline and Figure 2 represents sample outputs. Using a supervised machine

100 learning approach, we first trained our algorithms to identify each HCP parcel using network

101 connectivity from a normative dataset. Then, we applied our machine to identify the most

102 appropriate HCP parcels in brains after supratentorial tumour surgery based on the same input

103 imaging data. To our knowledge, this approach is unique in that previous studies have resolved

104 this issue by applying the HCP parcellation derived from healthy brains without any adjustment

105 to cortical topology.

106

\section{Comparative Connectome Analyses}

To gain additional insight into network connectivity, we processed $n=300 \mathrm{HCP}$ connectomes to serve as a reference of healthy canonical brain network organisation. Using this

110 normative data, we qualitatively compared healthy networks to those observed in patients with

111 lesions in particular areas. For example, we compared the normative visual areas to a patient

112 with hemianopia (Figure 3a) or normative language network topology with that of a patient with

113 aphasia (Figure 3b). This intra-network analysis enabled us to perform a hypothesis-driven

114 neuro-navigated rTMS target selection.

\section{rTMS treatment paradigm}

The rTMS treatment was initiated within 1-2 weeks after standard awake glioma surgery.

118 We utilized theta burst stimulation (TBS) protocols in all patients. Details of the TMS protocol

119 and rationale available in the SI. We performed treatment five times per day over five

120 consecutive days. In between TMS sessions, patients underwent rehabilitative therapy. 
medRxiv preprint doi: https://doi.org/10.1101/2021.07.27.21260088; this version posted August 4, 2021. The copyright holder for this preprint (which was not certified by peer review) is the author/funder, who has granted medRxiv a license to display the preprint in perpetuity.

All rights reserved. No reuse allowed without permission.

\section{Complications and Adverse Events}

All complications and side effects were noted after each rTMS session and one-week post-treatment. Seizures were defined as any observable seizure or possible seizure-like activity during the course of treatment. Neurological complications included any new or worsening of

126 neurological dysfunction measured by the ART and MRC Motor scale.

\section{Results}

Preliminary safety and recruitment data regarding rTMS treatment in neurosurgical patients.

We successfully recruited 31/34 (91\%) patients within two-weeks after glioma surgery and treated them with rTMS. The median participant age was 58 years with 20 females and 14 males. 30 patients had WHO grade II-IV gliomas, while four patients had low grade gliomas. Of all the participants, $n=23$ began rTMS therapy within a week of surgery, and $n=31$ began within

1342 weeks of surgery. The remaining 3 participants underwent treatment at 2 months, 4 months and

13512 months and excluded in the recruitment rate citing logistic concerns. In total, 31 participants

136 completed all planned treatment sessions with one participant missing one rTMS session due to a

137 rehabilitation bed becoming available the day of their last scheduled treatment. No participant

138 stopped therapy due to treatment intolerance. In 21 participants with a motor deficit, rTMS was

139 applied to the sensorimotor network with an improvement noted in 19 patients after one-week

140 following the last TMS session. In 13 participants with a language deficit, rTMS was applied to

141 the frontoparietal network with an improvement in 12 patients after one-week of the final

142 stimulation session.

Safety and preliminary efficacy of rTMS in neurosurgical patients

No participants reported any general or partial seizures or seizure-like events during the 147 course of treatment and follow-up. Four patients reported transient headaches which resolved at 148 the end of each individual session. Light headedness $(n=1)$ and nausea $(n=1)$ was also reported 149 but resolved before the start of the next session. Transient tingling was reported at the site of 150 stimulation during stimulation onset, but also resolved immediately. These results are consistent 151 with well-documented side-effects during rTMS of non-craniotomy patients ${ }^{[13,16,22]}$. We noted 
152 no worsening of neurological deficits and no other obvious side effects. The Supplemental

153 Digital Content is a video of a typical procedure; the participants consented to publication of

154 his/her image. Two brief clinical vignettes are presented below.

\section{Clinical Vignettes}

Case 1:

A woman (age 60-65) with a left parietal glioblastoma presented with preoperative aphasia and near complete hemiplegia. Following resection, she developed complete expressive

161 aphasia and right hemiplegia. Connectome analyses revealed that her sensorimotor networks

162 were fragmented as two independent parcellations, likely due to the destruction of the callosal

163 fibers. Specifically, the injured side demonstrated satellite areas anterior to the dysfunctional

164 sensorimotor networks (Figure 4). Additionally, the left frontoparietal network revealed a clear

165 component of Broca's area, area 55b, and an SMA component. However, the temporal

166 component appeared to be less organized, appearing abnormal compared to normative data.

167 Thus, to potentially enhance functional recovery and address both delocalised networks, we

168 sought to select a stimulation target that would lead to enhanced network recruitment. (cTBS) to both the middle of the right sensorimotor network and the posterior frontal component

172 of the right frontoparietal network (both targets treated once per day). We then performed

173 intermittent TBS (iTBS) to the areas of scattered activation in the posterior left temporal lobe

174 and the areas near the abnormal sensorimotor regions. This treatment was well tolerated, and by

175 the end of the treatment, the patient was able to ambulate with a cane and speak in full sentences.

176 There were no serious complications, however, she had some persistent arm weakness.

\section{Case 2:}

A man (70-75 years of age) with a posterior left insular glioblastoma had moderate pre-

180 operative expressive aphasia that persisted post-surgery. Connectome analyses demonstrated

181 that his posterior temporal region was appropriately organized but did not co-activate within the

182 same network as Broca's area (Figure 5) [6]. Thus, we hypothesized that this was the result of 
medRxiv preprint doi: https://doi.org/10.1101/2021.07.27.21260088; this version posted August 4, 2021. The copyright holder for this preprint

(which was not certified by peer review) is the author/funder, who has granted medRxiv a license to display the preprint in perpetuity.

All rights reserved. No reuse allowed without permission.

183 inactivation of the arcuate fasciculus fibers by the tumor or related to oedema. We also noted that

184 he was recruiting the right analog of Broca's area, as both regions were functionally co-activated.

185 As a result, we chose to perform accelerated (spaced-delivery of stimulation ${ }^{[23]}$ ) iTBS to the left

186 posterior temporal site to enhance the recruitment of additional connections for speech

187 improvement. This treatment began on post-operative day four. At the end day five of rTMS, his

188 speech markedly improved with no complications to report. Nevertheless, he persisted with

189 residual paraphasia after his therapy.

191 Discussion

In this study, we demonstrate the safety of rTMS post-craniotomy with the goal of

193 promoting functional recovery. Specifically, we demonstrate that no seizures were induced in 31

194 patients post-craniotomy and transient side effects were reported in 6 patients. This work

195 complements safety data from dozens of rTMS studies completed in non-craniotomy individuals

$196[24,25]$. Despite the uncontrolled and open-label nature of the study, we cautiously interpret that

197 rTMS can potentially facilitate functional recovery post-craniotomy.

198 Similar results have been illustrated in acute and chronic stroke patients suggesting the

199 possible role of TMS as a therapeutic modality for a variety of clinical conditions to facilitate

200 motor and language improvement ${ }^{[14]}$. Given the widely demonstrated safety profile of TMS, it

201 would be a disservice not to further investigate the efficacy of technology to optimize post-

202 surgical clinical outcomes. To fully harness interventional neurorehabilitation's potential for

203 neuro-oncological care, additional research is required in two areas: target engagement and

204 simulation protocol. Here, we elucidate the role of individualized TMS in standard inpatient

205 rehabilitation and discuss implications for future study on rTMS to optimize clinical outcomes.

206

\section{Importance of Target Engagement and Stimulation Protocol}

208 There is a growing body of evidence suggesting that effective TMS targeting is critical

209 for success. For example, using image guidance to target rTMS improves efficacy ${ }^{[26]}$.

210 Furthermore, targeting brain networks affected by disease-related processes is crucial for

211 functional improvement. Recently, Momi and colleagues delivered TMS pulses to two

212 frontoparietal nodes (prefrontal and parietal) to enhance fluid intelligence tasks ${ }^{[22]}$ - adding

213 another research consideration on multi-nodal, rather than uni-nodal, stimulation. In addition, it 
214 is likely that different patients with the same clinical deficit may need different target(s) ${ }^{\text {[27] }}$

215 Hence, there are many ways to interpret these observations, however, we advocate establishing

216 "the right target for the right patient" as being critical to successful interventional rehabilitation.

Similar to target engagement, stimulation protocol is another important variable to

219 consider. There are numerous different TMS protocols available for use. However, TBS

220 protocols are better suited for neurosurgical patients. First, the lower stimulus intensities used in

221 TBS likely have a lower seizure risk ${ }^{[28]}$. Second, TBS protocols achieve similar effects with

222 shorter treatment times (typically 8 minutes per session) compared to standard 30 minutes with

$22310 \mathrm{~Hz}$ TMS protocols. This enables the use of accelerated protocols (spaced-delivery of

224 stimulation sessions ${ }^{[23]}$ ) which are useful in treating patients in a subacute paradigm. Finally,

225 the stimulation effects of TBS is believed to last 45-60 mins which may fit better when

226 coordinating inter-session rehabilitation ${ }^{[29,30]}$. Our view is that while seizures are a concern,

227 given our clinical experience in managing this problem concomitant with the low occurrence rate

228 of this complication, there is now sufficient evidence to justify offering neurosurgical patients

229 rTMS.

230

\section{Connectome-based Stimulation for Cognitive Rehabilitation}

In this study, we primarily focused on ameliorating motor and language deficits post-

233 surgery in glioma patients. However, many patients experience cognitive deficits post-surgery

234 and there are no clear guidelines on how to help these patients. The multiple demand (MD)

235 system is a domain-general cognitive control network that acts as a skeleton for executing

236 cognitive tasks [3,7]. Systematically studying this system and the implications of its removal

237 during surgery would be useful for predicting post-operative cognitive trajectories ${ }^{[31]}$. More

238 broadly, despite motor or language deficits in our cohort, the qualitative fundamental motivation

239 to rehab greatly varied between our individuals ${ }^{[32]}$. An increasing line of evidence suggests that

240 increasing the motivation to expend cognitive effort, rather than enhancing cognitive networks

241 themselves, would be more effective in bolstering goal-directed behaviour ${ }^{[33]}$. Thus, if

242 frontostriatal circuitry can be mapped and effectively modulated post-craniotomy, this would be

243 a significant advancement and become important for other areas of neurosurgery, such as limbic

244 surgery ${ }^{[34]}$. 
medRxiv preprint doi: https://doi.org/10.1101/2021.07.27.21260088; this version posted August 4, 2021. The copyright holder for this preprint (which was not certified by peer review) is the author/funder, who has granted medRxiv a license to display the preprint in perpetuity.

All rights reserved. No reuse allowed without permission.

\section{Establishing a TMS Clinic for a Neurosurgical Practice}

While rTMS is a well-developed field with standard techniques, treating post-craniotomy patients posed some unique challenges. First, the benefit of TMS early in the post-operative period appears important ${ }^{[35]}$. Due to logistical issues, not all patients were able to return within one week of surgery and returned within 14 days. However, there are important limits to early TMS intervention in neurosurgical patients. Chiefly, given the small risk of inducing a seizure, patients with significant swelling and/or midline shifts are not optimal candidates for rTMS.

253 Furthermore, patients with seizure history due to a tumour should be excluded from TMS until 254 more evidence of its safety in these circumstances are available. Despite these issues, we now

255 feel rTMS can be safely performed as part of standard inpatient rehabilitation.

\section{rTMS Therapy for Stroke and Surgery}

The literature on the role of TMS in motor and language functional recovery is wellestablished in stroke patients, providing most of our insight into the current benefits and limitations of this therapeutic modality. Thus, certain themes from stroke neurology may cautiously applied to neuro-oncological patients to guide therapeutic stimulation. For example, cerebral inflammation and angiogenesis are two of multiple overlapping processes between glioma surgery and cerebral ischemic stroke pathways [36]. A recent meta-analysis on 841 patients across 20 randomized controlled trials (RCTs) demonstrates that rTMS is beneficial to the treatment of post-stroke hemiplegia, especially in: lower limb functioning, grip strength, and attenuating stroke severity [15]. Interestingly, cortical reorganization can be observed between primary motor and secondary motor cortices in stroke patients to facilitate improved motor

268 functioning, leading authors to suggest the need for future customized TMS applications based

269 on the newly activated cortical pathways in these stroke patients [37]. Similar cortical re-

270 organization has also been demonstrated to facilitate language functioning following ischemic

271 stroke [38]. Thus, following iatrogenic injury due to tumor resection, it is likely that similar

272 cortical pathways responsible for motor functioning and motor learning can also be strengthened

273 with rTMS given they too demonstrate plasticity following tumor growth $[39,40]$. 
rTMS likely provides additional benefits in many patients following tumor resection, while being precluded from some stroke patients, due to certain temporal factors which are known to affect plasticity. For instance, certain gliomas may cause slower changes in cerebral connections and therefore affect connectome plasticity differently compared to acutely damaging stroke lesions[39]. Unfortunately, acute brain damage due to stroke often causes more localized neuronal cell death and subsequent unimodular cortical organization [8]. This type of damage may demonstrate less capacity for cerebral reorganisation compared to slow growing tumors (ie,

282 low-grade gliomas, LGG), which disrupts greater amounts of cortex but provides more opportunity for functional reorganization possibly due to less abrupt, initial neuronal death [8]. Thus, even more importantly in tumor patients, individualized connectomic approaches can be implemented before rTMS to identify any on-going network reorganization occurring from the lesion and ultimately facilitate motor and language preservation or compensation. This would enable an updated understanding of network connectivity which can be applied to effectively target and strengthen formerly silent polysynaptic cortical pathways following tumor resection ${ }^{[14,40]}$. Indeed, our study shows that intra-network analyses can safely enable hypothesis-driven neuro-navigated rTMS target selections.

While the applications of TMS between stroke and surgical patients have not been compared, their respective methods may differ and therefore preclude certain benefits which have been demonstrated specifically in ischemic stroke patients. Unfortunately, no studies were able to be identified which looked at post-surgical rehabilitation utilizing individualized rTMS treatment. Most surgical studies incorporating TMS have confined their uses to intra-operative functional mapping (ie, language mapping) [41]. The only post-surgical application of rTMS identified investigated its ability to reduce pain following gastric bypass surgery [42].

299 Nonetheless, in stroke patients, the effects produced by TMS correlates with the specific TMS 300 protocol applied ${ }^{[14]}$. Specifically, a TBS protocol of $50 \mathrm{~Hz}$ with $80 \%$ of active motor threshold 301 intensity is believed to modulate motor learning through effects similar long-term potentiation 302 (LTP) and long-term depression (LTD), and has been applied in numerous stroke trials ${ }^{[14,43]}$. Our 303 study utilized bursts of 3 pulses delivered with a repetition rate of $50 \mathrm{~Hz}$ at $80 \%$ of motor 304 threshold for 400 continuous (cTBS) or intermittent (iTBS) trains. Still, while our protocol is similar to those described for ischemic stroke patients and we too observed improvements in 
language and motor functioning, possible differences in neuroplasticity as identified by neurocomputational models between glioma and stroke patients suggests that specific rTMS protocols may produce different cortical changes and subsequent functional recovery based on the type cortical injury.

\section{Future Directions for Interventional Neurorehabilitation}

With the rapid advancements made in imaging and stimulation technologies [32], the

313 future is bright to continuously evolve the concept of interventional neurorehabilitation for

314 neuro-oncology. Importantly, pre-operatively predicting which patients will require post-

315 operative interventional support or predicting which patients will best respond to and benefit

316 from rTMS as a therapeutic adjunct would be highly valuable. rTMS treatment causes changes in

317 electroencephalographic (EEG) assessed- functional connectivity (FC) that correlates well with

318 clinical outcomes in certain patients. Thus, EEG-FC measures can provide accurate, early,

319 biomarkers to guide personalized clinical decisions for long-term rTMS treatments or not,

320 possibly saving hospital costs while improving patient quality of life over time [44]. A machine

321 learning algorithm can accurately predict (83\%) which patients with major depressive disorder

322 (MDD) will respond to long-term rTMS treatment in just the first rTMS session [45]. Similar

323 studies have not been extended into the perioperative setting, but numerous studies have

324 established the role of TMS and fMRI data as biomarkers to predict motor recovery following

325 stroke, warranting further investigation following neuro-oncological resection [46,47]. Lastly,

326 interventional neurorehabilitation can also be utilised in other patient populations following

327 stroke-induced pain or movement disorders [48-50]

328 With rising open science and international collaborative efforts, prospective registrybased clinical studies could be employed to overcome heterogeneous glioma populations and derive more practical outcomes.

\section{Study Limitations}

This study, however, is not without key limitations. First, the uncontrolled and early-

334 nature of our intervention raises the possibility whether these patients would have improved

335 without treatment. Now that we have established the TMS safety profile and recruitment rate for 336 this complex patient population, future trials should employ prospective, randomised, double- 
blinded active- and sham-controlled TMS to determine the efficacy of improving recovery trajectories. While glioma patients are highly heterogenous given different tumors, different degrees of cortical reorganisation, and different resections completed, strict inclusion criteria and multi-site collaborations can overcome such limitations. An alternative approach would be to conduct large-scale international prospective registry-based studies. Finally, we did not acquire

342 long-term outcome data in these patients. Nevertheless, qualitatively, all patients reported they

343 would undergo TMS therapy again and found that it assisted with their rehabilitative efforts.

344 Despite these limitations, our primary aim was to establish the safety profile and recruitment rate for TMS post-craniotomy.

\section{Conclusion}

In conclusion, we present a proof-of-concept of 'interventional neurorehabilitation' for neuro-oncological clinicians to take charge in driving cortical reorganisation and functional recovery. Specifically, we demonstrate the safety profile and recruitment rate for connectomebased TMS acutely post-surgery for glioma patients. Given the clear enthusiasm from our patients, we believe that TMS treatment is of low-risk, well-tolerated, and could be of immense therapeutic benefit.

\section{REFERENCES}

3581 Bullmore, E. \& Sporns, O. Complex brain networks: graph theoretical analysis of structural and functional systems. Nat Rev Neurosci 10, 186-198, doi:10.1038/nrn2575

3612 Sporns, O. (The MIT Press, 2010).

3623 Duncan, J. The multiple-demand (MD) system of the primate brain: mental programs for intelligent behaviour. Trends Cogn Sci 14, 172-179, doi:10.1016/j.tics.2010.01.004 (2010).

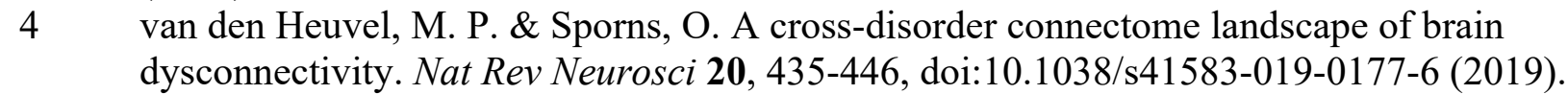
Tumors on Functional Complexity and Network Robustness: A Prospective Cohort Study. Neurosurgery 84, 1201-1213, doi:10.1093/neuros/nyy378 (2019). 
3737 Poologaindran, Anujan, Stephen R. Lowe, and Michael E. Sughrue. "The cortical organization of language: distilling human connectome insights for supratentorial neurosurgery." Journal of Neurosurgery 134.6 (2020): 1959-1966. Keidel, J. L., Welbourne, S. R. \& Lambon Ralph, M. A. Solving the paradox of the equipotential and modular brain: A neurocomputational model of stroke vs. slowgrowing glioma. Neuropsychologia 48, 1716-1724, doi:https://doi.org/10.1016/j.neuropsychologia.2010.02.019 (2010).

3809 Cui, Z. et al. Individual Variation in Functional Topography of Association Networks in Youth. Neuron 106, 340-353 e348, doi:10.1016/j.neuron.2020.01.029 (2020). Finn, E. S. et al. Functional connectome fingerprinting: identifying individuals using patterns of brain connectivity.

11 Beaty, R. E. et al. Robust prediction of individual creative ability from brain functional connectivity. Proc Natl Acad Sci U S A 115, 1087-1092, doi:10.1073/pnas.1713532115 (2018).

12 Grossman, N. et al. Noninvasive Deep Brain Stimulation via Temporally Interfering Electric Fields. Cell 169, 1029-1041 e1016, doi:10.1016/j.cell.2017.05.024 (2017). Treatment of Major Depressive Episodes: A Systematic Review With Network Metaanalysis. JAMA Psychiatry 74, 143-152, doi:10.1001/jamapsychiatry.2016.3644 (2017). Hoyer, E. H. \& Celnik, P. A. Understanding and enhancing motor recovery after stroke using transcranial magnetic stimulation. Restor Neurol Neurosci 29, 395-409, doi:10.3233/RNN-2011-0611 (2011). He, Y., Li K Fau - Chen, Q., Chen Q Fau - Yin, J., Yin J Fau - Bai, D. \& Bai, D. Repetitive Transcranial Magnetic Stimulation on Motor Recovery for Patients With Stroke: A PRISMA Compliant Systematic Review and Meta-analysis. transcranial magnetic stimulation (rTMS).

17 Brain, M. R. C. G. o. Aids to the Examiantion of the Peripheral Nervous System. (Balliere Tindall, 1986).

18 Azuar, C. et al. The Aphasia Rapid Test: an NIHSS-like aphasia test. J Neurol 260, 21102117, doi:10.1007/s00415-013-6943-x (2013).

19 Romero-Garcia, R. et al. Practical Application of Networks in Neurosurgery: Combined 3-Dimensional Printing, Neuronavigation, and Preoperative Surgical Planning. World Neurosurg 137, e126-e137, doi:10.1016/j.wneu.2020.01.085 (2020). Connectional Anatomy of Human Brain Networks. Oper Neurosurg (Hagerstown) 15, S470-S480, doi:10.1093/ons/opy272 (2018).

21 Glasser, M. F. et al. A multi-modal parcellation of human cerebral cortex. Nature 536, 171-178, doi:10.1038/nature18933 (2016).

22 Momi, D. et al. Cognitive Enhancement via Network-Targeted Cortico-cortical Associative Brain Stimulation. Cereb Cortex 30, 1516-1527, doi:10.1093/cercor/bhz182 (2020).

23 Cole, E. J. et al. Stanford Accelerated Intelligent Neuromodulation Therapy for Treatment-Resistant Depression. Am J Psychiatry 177, 716-726, doi:10.1176/appi.ajp.2019.19070720 (2020). 
24 Di Iorio, R. \& Rossini, P. M. in Navigated Transcranial Magnetic Stimulation in Neurosurgery (ed Sandro M. Krieg) 67-83 (Springer International Publishing, 2017).

25 Rossi, S., Hallett, M., Rossini, P. M. \& Pascual-Leone, A. Safety, ethical considerations,

26 Ahdab, R., Ayache, S. S., Brugieres, P., Goujon, C. \& Lefaucheur, J. P. Comparison of

29 Wischnewski, M. \& Schutter, D. J. Efficacy and Time Course of Theta Burst Stimulation in Healthy Humans. Brain Stimul 8, 685-692, doi:10.1016/j.brs.2015.03.004 (2015).

31 Briggs, R. G. et al. The Frontal Aslant Tract and Supplementary Motor Area Syndrome:

27 Drysdale, A. T. et al. Erratum: Resting-state connectivity biomarkers define neurophysiological subtypes of depression. Nat Med 23, 264, doi:10.1038/nm0217-264d (2017).

28 Oberman, L., Edwards, D., Eldaief, M. \& Pascual-Leone, A. Safety of theta burst transcranial magnetic stimulation: a systematic review of the literature. J Clin Neurophysiol 28, 67-74, doi:10.1097/WNP.0b013e318205135f (2011).

32 Satterthwaite, T. D., Xia, C. H. \& Bassett, D. S. Personalized Neuroscience: Common and Individual-Specific Features in Functional Brain Networks. Neuron 98, 243-245, doi:https://doi.org/10.1016/j.neuron.2018.04.007 (2018).

33 Westbrook, A. et al. Dopamine promotes cognitive effort by biasing the benefits versus costs of cognitive work. Science 367, 1362-1366, doi:10.1126/science.aaz5891 (2020).

34 Hurwitz, T. A., Honey, C. R., McLeod, K. R., Poologaindran, A. \& Kuan, A. J. Hypoactivity in the Paraterminal Gyrus Following Bilateral Anterior Capsulotomy. Can J Psychiatry 65, 46-55, doi:10.1177/0706743719874181 (2020).

35 Volz, L. J. et al. Shaping Early Reorganization of Neural Networks Promotes Motor Function after Stroke. Cereb Cortex 26, 2882-2894, doi:10.1093/cercor/bhw034 (2016).

36 Ghosh, M. K., Chakraborty, D., Sarkar, S., Bhowmik, A. \& Basu, M. The interrelationship between cerebral ischemic stroke and glioma: a comprehensive study of recent reports. Signal Transduction and Targeted Therapy 4, 42, doi:10.1038/s41392019-0075-4 (2019).

37 Sharma, N., Baron, J.-C. \& Rowe, J. B. Motor imagery after stroke: relating outcome to motor network connectivity. Ann Neurol 66, 604-616, doi:10.1002/ana.21810 (2009).

38 Hamilton, R., Keenan Jp Fau - Catala, M., Catala M Fau - Pascual-Leone, A. \& PascualLeone, A. Alexia for Braille following bilateral occipital stroke in an early blind woman.

39 Kong, N. W., Gibb, W. R. \& Tate, M. C. Neuroplasticity: Insights from Patients Harboring Gliomas. Neural Plast 2016, 2365063-2365063, doi:10.1155/2016/2365063 (2016).

40 Kawashima, A. et al. Plastic reshaping of cortical language areas evaluated by navigated transcranial magnetic stimulation in a surgical case of glioblastoma multiforme. 
$46441 \quad$ Ille, S. et al. Resection of highly language-eloquent brain lesions based purely on rTMS language mapping without awake surgery.

42 Borckardt Jj Fau - Reeves, S. T. et al. Fast left prefrontal rTMS reduces post-gastric bypass surgery pain: findings from a large-scale, double-blind, sham-controlled clinical trial.

43 Huang, Y. Z., Edwards Mj Fau - Rounis, E., Rounis E Fau - Bhatia, K. P., Bhatia Kp Fau - Rothwell, J. C. \& Rothwell, J. C. Theta burst stimulation of the human motor cortex.

44 Voigt, J., Carpenter, L. \& Leuchter, A. Cost effectiveness analysis comparing repetitive transcranial magnetic stimulation to antidepressant medications after a first treatment failure for major depressive disorder in newly diagnosed patients - A lifetime analysis. PLoS One 12, e0186950-e0186950, doi:10.1371/journal.pone.0186950 (2017).

45 Corlier, J. et al. Changes in Functional Connectivity Predict Outcome of Repetitive Transcranial Magnetic Stimulation Treatment of Major Depressive Disorder. Cerebral cortex (New York, N.Y. : 1991) 29, 4958-4967, doi:10.1093/cercor/bhz035 (2019).

46 Boyd, L. A. et al. Biomarkers of stroke recovery: Consensus-based core recommendations from the Stroke Recovery and Rehabilitation Roundtable. Int J Stroke 12, 480-493, doi:10.1177/1747493017714176 (2017).

47 Kim, B. \& Winstein, C. Can Neurological Biomarkers of Brain Impairment Be Used to Predict Poststroke Motor Recovery? A Systematic Review.

48 Ren, H. et al. Application of Structural and Functional Connectome Mismatch for Classification and Individualized Therapy in Alzheimer Disease. Frontiers in Public Health 8, 720 (2020).

49 Ivanishvili Z, Poologaindran A, Honey CR. Cyclization of motor cortex stimulation for neuropathic pain: a prospective, randomized, blinded trial. Neuromodulation. 2017;20(5):497-503.

Acknowledgements: AP is funded through a Turing Doctoral Scholarship from the Alan Turing supported by a Guarantors of Brain Fellowship.

Financial Disclosures: IMY is an employee of Cingulum Health. MES is the chief medical

499 officer of Omniscient and a shareholder of Cingulum Health. CT is a consultant for Aesculap.

500 All other authors report no conflict of interest related to this study. 
medRxiv preprint doi: https://doi.org/10.1101/2021.07.27.21260088; this version posted August 4, 2021. The copyright holder for this preprint (which was not certified by peer review) is the author/funder, who has granted medRxiv a license to display the preprint in perpetuity.

All rights reserved. No reuse allowed without permission.

503 Author Contributions: Conceptualization, AP, IMY and MES; Data curation, CP, IMY;

504 Formal analysis, AP, IMY.; Methodology, AP, RGB, MES; Project administration, IMY, CT.

505 and M.E.S.; Software SAA, KC, RRG; Writing — original draft, AP.; Writing - review and

506 editing, NBD, RRG, JS, MES.

\section{FIGURE LEGENDS}

512 Figure 1: The connectome construction pipeline used in this study. A) A standard Glasser atlas

513 was established using 300 healthy individuals from the Human Connectome Project (HCP). A

514 supervised machine learning algorithm was employed to recognise connectivity patterns for each

515 of the $360 \mathrm{HCP}$ parcels in a healthy cohort. B) Using diffusion sequences, we applied

516 constrained spherical deconvolution (CSD) tractography to our patient cohort. Using these

517 images, our algorithm was applied to recognise and adjust the locations of HCP parcels in highly

518 atypical brains. C) After establishing maximal likely structural connectivity, we used this data to

519 inform and constrain functional connectivity using resting-state fMRI. D) Finally, structural and

520 functional anomaly matrices were generated to compare network connectivity differences (i.e.

521 language) between our patient and a normative atlas. Adopted with permission from Reference $522[48]$. 


\section{Connectome Construction Pipeline}

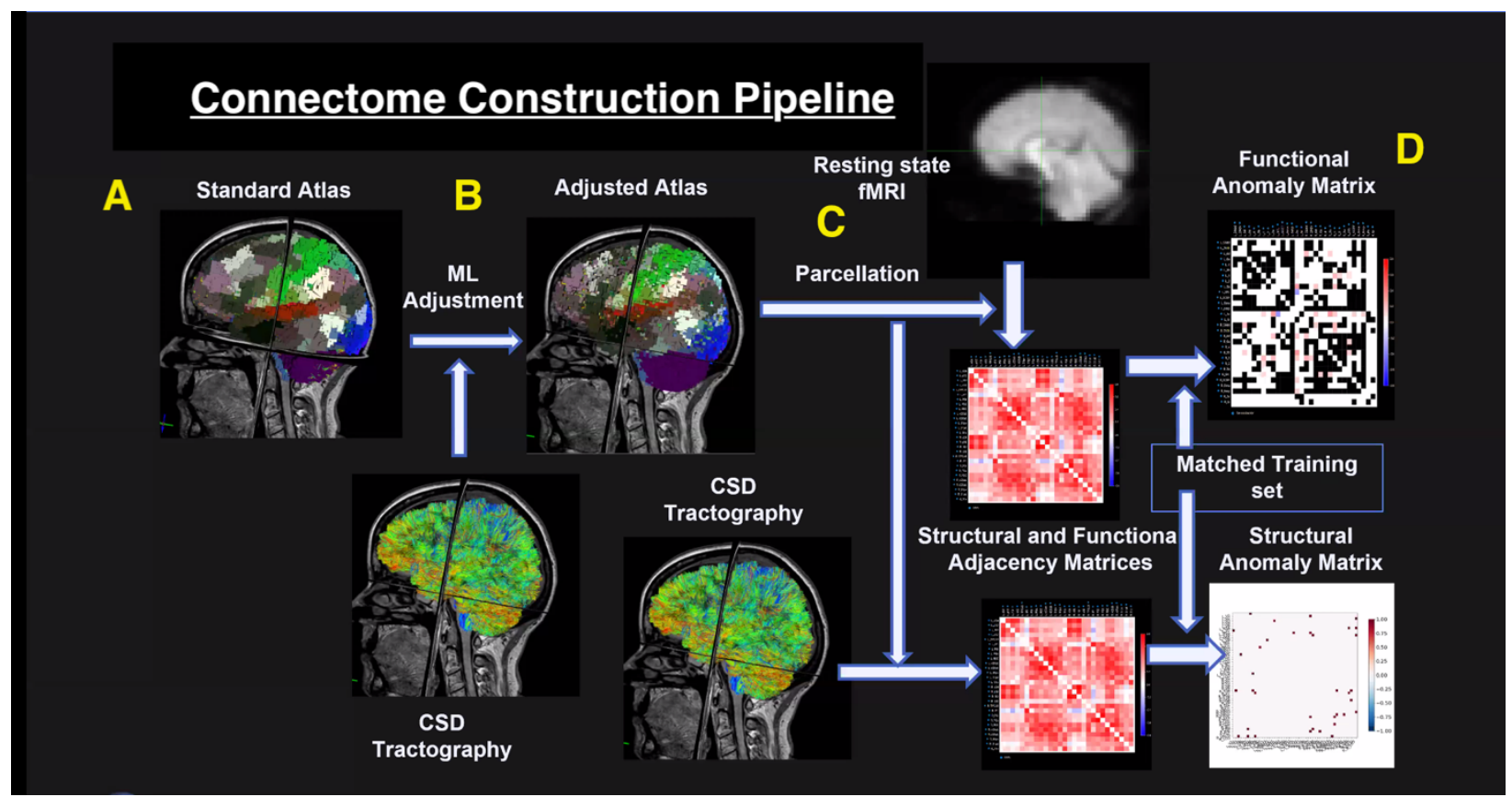

527 Figure 2: Demonstration of proprietary machine learning algorithm (Omniscient) that assigns

528 parcellations to very distorted brains. Patient with a frontal lobe GBM and resected regions

529 resulting in total anterior brain shift. Figure 2a displays the modified location of the caudate

530 nucleus and the putamen. Figure $2 \mathrm{~b}$ displays the modified location of the GP. Figure 2c displays

531 the modified location of the basal forebrain. Figure $2 \mathrm{~d}$ displays the modified location of right $55 \mathrm{~b}$

532 parcellation. Figure 2e displays the modified location of the right PBelt. This allows for the

533 creation of a connectivity matrix of any brain despite . 
medRxiv preprint doi: https://doi.org/10.1101/2021.07.27.21260088; this version posted August 4, 2021. The copyright holder for this preprint (which was not certified by peer review) is the author/funder, who has granted medRxiv a license to display the preprint in perpetuity.

All rights reserved. No reuse allowed without permission.
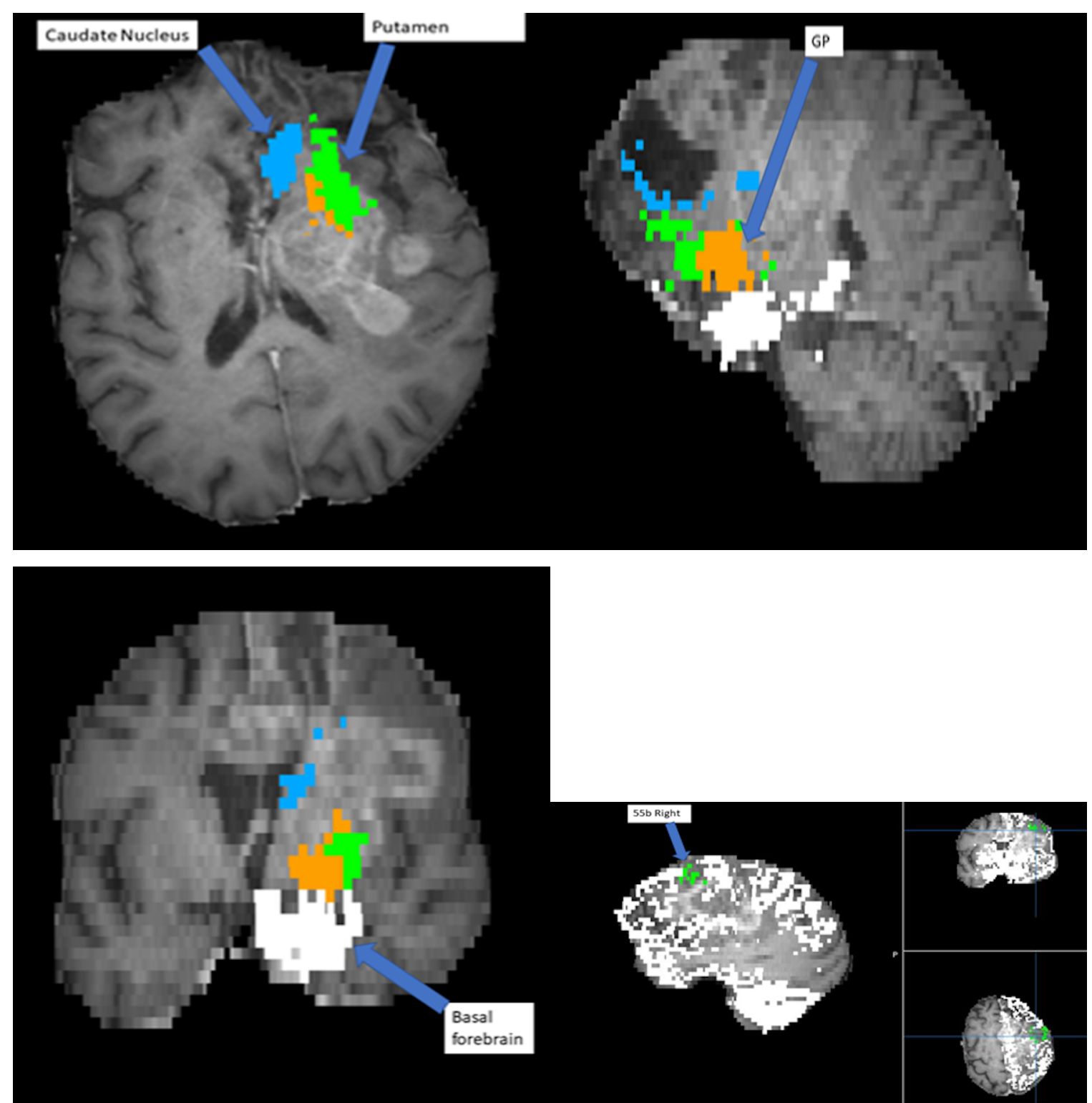

536

537

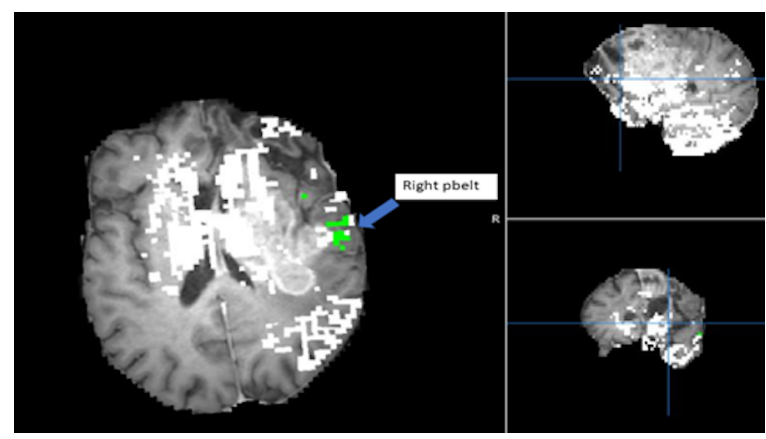


541 Figure 3: 3a compares connectivity matrices of the left and right visual networks in a patient

542 with hemianopia. The left visual network is dotted mostly blue, which means that areas of the

543 visual system are not well synchronized to one another. By comparison, the right visual network

544 displays strong intra-network connectivity. Figure $3 \mathrm{~b}$ compares the connectivity matrix of the

545 language area of a healthy control on the left with the language area connectivity of an aphasic

546 patient on the right. This aphasic matrix has the parcellations within the language system

547 anticorrelated, therefore, predominantly blue, suggestion loss of connectivity within the language

548 network. Note that columns 55b, 45 and STSdp are blue representing that they are isolated. We

549 hypothesized that this is in part due to problems with the superior longitudinal fasciculus/ arcuate

550 fasciculus system which links different components of the language system ${ }^{7}$. Conducting

551 connectomics analysis by comparing connectivity matrices enables us to generate potential

552 targets for TMS treatment.

553
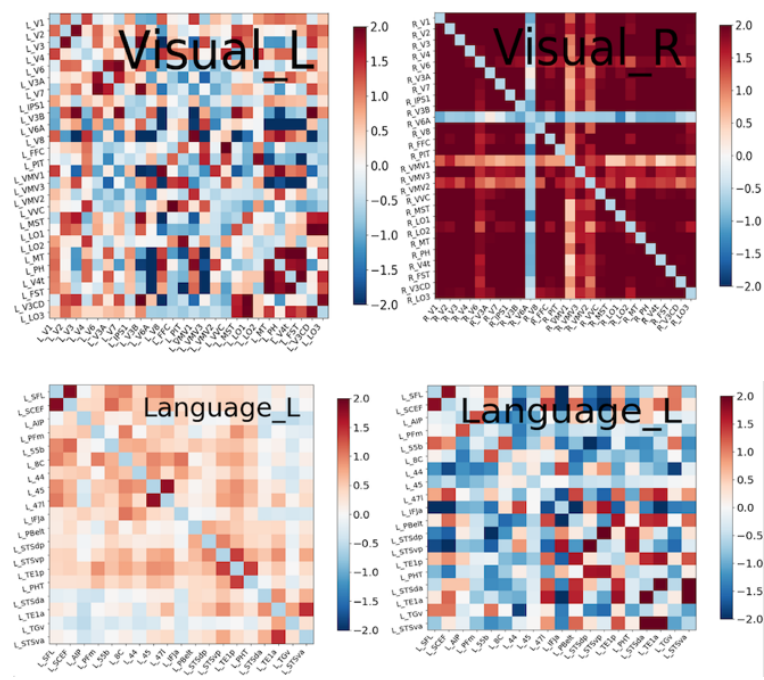

Figure 4: TMS strategy for patient presenting with aphasia and near complete hemiplegia secondary to glioblastoma. (a) Postoperative MRI of patient demonstrating resection cavity. (b)

560 Independent right sided (green) and left sided (orange) sensorimotor networks. Although

561 presented on the same image, these networks appeared as separate networks on connectomic 562 analysis. The anterior satellite areas in the left (orange) dysfunctional sensorimotor network. (c)

563 Left frontoparietal network demonstrating clear Broca's area and area 55b. The temporal 
medRxiv preprint doi: https://doi.org/10.1101/2021.07.27.21260088; this version posted August 4, 2021. The copyright holder for this preprint (which was not certified by peer review) is the author/funder, who has granted medRxiv a license to display the preprint in perpetuity.

All rights reserved. No reuse allowed without permission.

564 component of the network is disorganized. (d) cTBS was administered to both the middle of the

565 sensorimotor network and the right posterior frontal component of the right frontoparietal

566 network. (e) iTBS was administered to the disorganized temporal component of the left

567 frontoparietal network and the (f) anterior areas of the pathological left sensorimotor network.

568
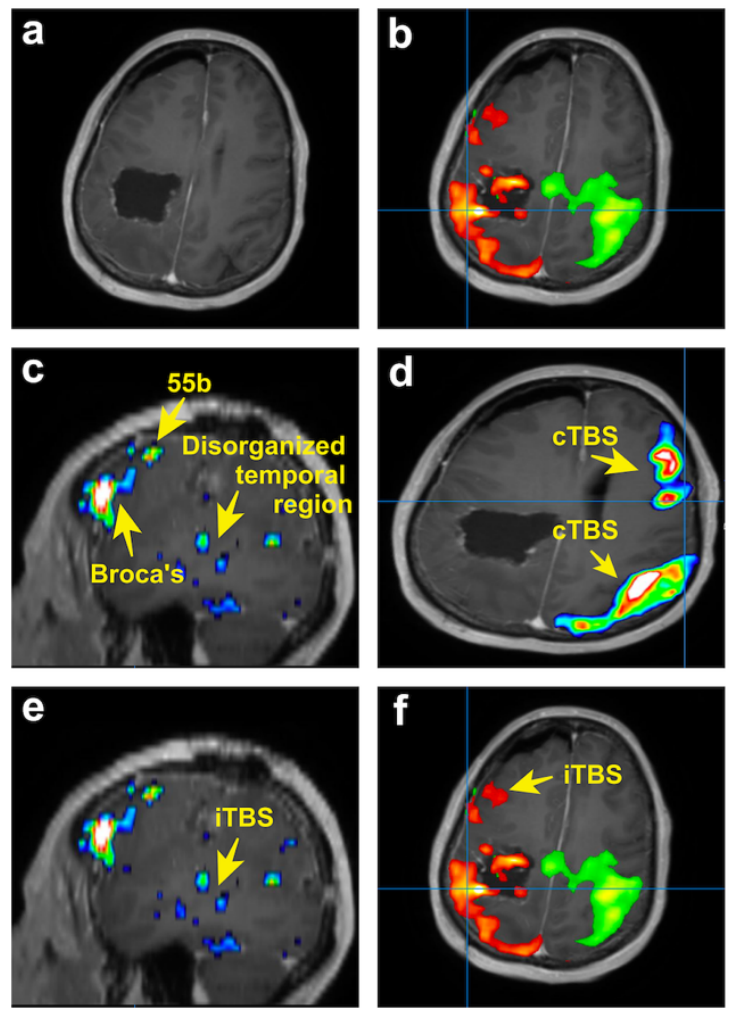

572 Figure 5: TMS strategy for patient presenting with moderate expressive aphasia secondary to

573 glioblastoma. (a) Preoperative MRI (left) demonstrating left insula glioblastoma and

574 postoperative MRI (right) demonstrating complete resection. (b) Network analysis demonstrating 575 a strongly organized posterior temporal region that is not in in the same network as Broca's area.

576 This was the area that was selected for treatment with iTBS. (c). Further network analysis

577 demonstrating Broca's area with bilateral representation that is not in the same network as the 578 posterior temporal region. 
medRxiv preprint doi: https://doi.org/10.1101/2021.07.27.21260088; this version posted August 4, 2021. The copyright holder for this preprint (which was not certified by peer review) is the author/funder, who has granted medRxiv a license to display the preprint in perpetuity.

All rights reserved. No reuse allowed without permission.
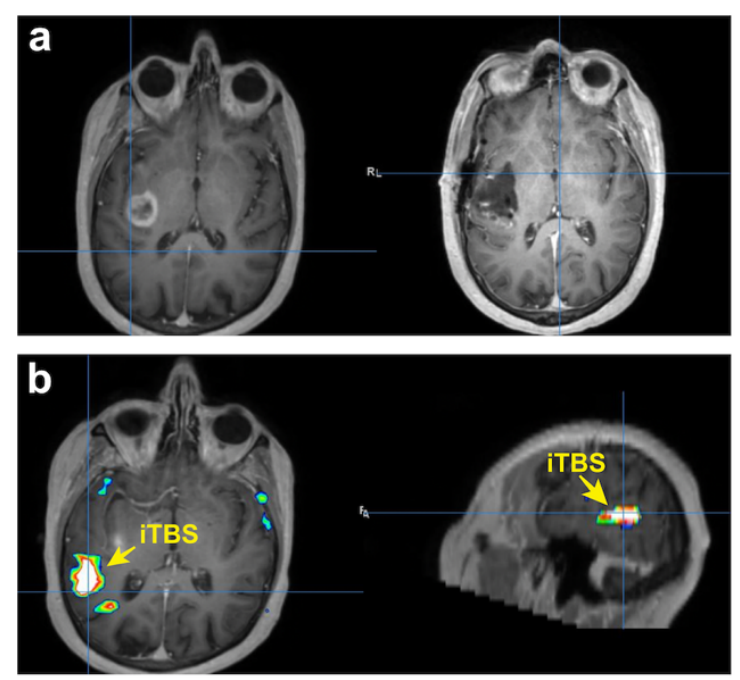

583 Supplemental Digital Content: This video expands on the TMS technique described in the

584 Methods. The video illustrates patient set-up, patient registration, measuring motor threshold, 585 and TMS treatment. The participants consented to publication of his/her image.

586 https://www.dropbox.com/s/prp7dm3h7bsgm62/TMS_Demonstration_Video.mp4?dl=0 